\title{
Different patterns of aortic wall elasticity in patients with Marfan syndrome: A noninvasive follow-up study
}

Daniela Baumgartner, MD, a Christian Baumgartner, $\mathrm{PhD},{ }^{\mathrm{b}}$ Elisabeth Schermer, MD, a Georg Engl, MD, ${ }^{a}$ Ulrich Schweigmann, MD, ${ }^{a}$ Gabor Mátyás, $\mathrm{PhD},{ }^{c, d}$ Beat Steinmann, $M D,{ }^{c}$ and Jörg Ingolf Stein, $\mathrm{MD}^{\mathrm{a}}$

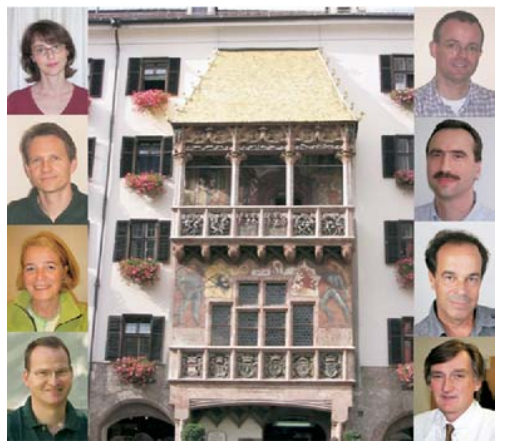

Golden Roof, Innsbruck, Austria. Left column (top-bottom): Drs D. Baumgartner, C.

Baumgartner, Schermer, and Engl; right column: Drs Schweigmann, Mátyás, Steinmann, and Stein
From the Department of Pediatric Cardiology, Innsbruck Medical University, Innsbruck, Austria, ${ }^{\text {a }}$ Research Group for Clinical Bioinformatics, Institute of Biomedical Engineering, University for Health Sciences, Medical Informatics and Technology Hall, Tyrol, Austria, ${ }^{\mathrm{b}}$ Division of Metabolism and Molecular Pediatrics, University Children's Hospital, Zurich, Switzerland, and Division of Medical Molecular Genetics and Gene Diagnostics, Institute of Medical Genetics, University of Zurich, Schwerzenbach, Switzerland. ${ }^{\mathrm{d}}$

D.B. and C.B. contributed equally to this work.

This study was supported by the Austrian Industrial Promotion Fund FFG (HITT-10 UMIT), the Wolfermann-Nägeli-Stiftung (Zurich), and the Swiss National Science Foundation (grant 3200-059 445/2; B.S.).

Received for publication April 22, 2006; revisions received June 20, 2006; accepted for publication July 7, 2006.

Address for reprints: Daniela Baumgartner, MD, Department of Pediatric Cardiology, Innsbruck Medical University, Anichstrasse 35, A 6020 Innsbruck, Austria (E-mail: daniela.baumgartner@uibk.ac.at).

J Thorac Cardiovasc Surg 2006;132:811-9

$0022-5223 / \$ 32.00$

Copyright (C) 2006 by The American Association for Thoracic Surgery

doi:10.1016/j.jtcvs.2006.07.001
Objective: Aortic complications determine the life expectancy of most patients with Marfan syndrome. To find out whether there is heterogenous aortic involvement among patients and, if there is, to characterize aortic patterns and response to long-term $\beta$-blocker therapy, we investigated aortic elastic properties before and during $\beta$-blocker treatment.

Methods: In 46 patients with Marfan syndrome (age, $17.4 \pm 11.1$ years) and 46 healthy control subjects, ascending and descending aortic elastic parameters were determined noninvasively before and after $39 \pm 16$ months of $\beta$-blocker treatment with atenolol.

Results: Aortic diameters and distensibility distinguished Marfan patients and controls with a sensitivity of $85 \%$ and a specificity of $87 \%$. Cluster analysis revealed 4 patterns of aortic phenotypic expression: (1) reduced ascending aortic elasticity (46\% of patients), (2) diminished ascending and descending aortic elasticity (17\%), (3) minimal alterations of ascending and descending aortic elasticity (20\%), and (4) reduced descending aortic elasticity $(17 \%)$. During follow-up, aortic elastic properties improved in $21(70 \%)$ of 30 patients and deteriorated in $9(30 \%)$ irrespective of $\beta$-blocker dosage. Improvement was observed in $100 \%$ of patients $(\mathrm{n}=7$; age, $5.3 \pm 4.2$ years) with end-diastolic aortic root diameters between 20 and $30 \mathrm{~mm}$ and in $61 \%$ of patients $(14 / 23$; age, $20.5 \pm 10.0$ years) with root diameters between 30 and $52 \mathrm{~mm}$.

Conclusions: Aortic elastic parameters distinguish between patients with Marfan syndrome and healthy controls and show the pattern of regional aortic involvement. Improvement or deterioration during follow-up can influence therapeutic decisions to prevent aortic dissection and rupture. Young age, small root diameter, and high distensibility are favorable prognostic factors.

$\mathrm{M}$ arfan syndrome (MFS) is an autosomal dominant connective tissue disorder caused by mutations in the gene encoding fibrillin 1 ( $F B N I)$, with highly variable clinical manifestations in the musculoskeletal, ocular, and cardiovascular systems. ${ }^{1,2}$ Recently, heterozygous mutations in the TGFBRI and TGFBR2 genes have been reported in MFS type 2 (MFS2), Loeys-Dietz aortic aneurysm syndrome, and familial thoracic aortic aneurysms and dissections (see review ${ }^{2}$ ). The leading cause of premature death is aortic dissection due to progressive aortic root dilation. ${ }^{3}$ Complications are not always predictable exclusively on the basis of aortic root dimensions. Notably, in a recent study of 268 MFS patients, almost 1 of every 3 aortic events (dissection or elective surgery) involved the distal aorta. ${ }^{4}$ Several studies, by using different techniques (cardiac catheterization, echocardiography, and magnetic resonance imaging), investigated aortic hemodynamic indexes such as impedance, wave reflections, compliance, pulse wave velocity, wall stiffness index, and distensibility. ${ }^{5-12}$ Nevertheless, the predictive power of these parameters has not been completely clari- 


\author{
Abbreviations and Acronyms \\ AscAo = ascending aorta \\ DescAo $=$ descending aorta \\ FBN1 = the gene encoding fibrillin 1 \\ MFS = Marfan syndrome \\ MSAI = maximum systolic area increase
}

fied, although some relations are known. ${ }^{10-12}$ Beta blockers have been shown to reduce the rate of aortic root dilation in both adolescent and adult patients with MFS, ${ }^{13}$ and there is evidence of improvement in children. ${ }^{14}$ However, a heterogenous response of patients to $\beta$-blockers depending on aortic diameter at the time of treatment induction was shown. ${ }^{15}$

The aim of our study was to find out whether there is heterogeneity of aortic involvement among patients and, if there is, to characterize different patterns of aortic involvement by means of a noninvasive method and to study aortic elastic properties before and during treatment with $\beta$-blockers, as well as to create a model that can serve either as a marker for the presence of MFS or as a noninvasive tool to assess aortic wall abnormalities during follow-up.

\section{Methods}

\section{Patients and Control Subjects}

Our study population consisted of 46 individuals (15 males and 31 females) with MFS according to the Ghent criteria. ${ }^{16}$ Age ranged between 7 months and 47 years (mean, $17.4 \pm 11.1$ years). Physical features were documented and graded according to the consensus of 2 physicians (D.B. and E.S.), according to a standardized protocol. Mutation analysis of the FBN1 gene was performed in 26 MFS patients as described elsewhere. ${ }^{12,17}$ Before the initial investigation, no patient received a $\beta$-blocker, angiotensinconverting enzyme inhibitor, or calcium antagonist or had a history of aortic dissection or aortic surgery. Of 32 patients ( 9 men and 23 women) available for follow-up investigations, 30 were treated orally with a $\beta$-blocker (atenolol $0.79 \pm 0.46 \mathrm{mg} \cdot \mathrm{kg}^{-1} \cdot \mathrm{d}^{-1}$ ) because of aortic dilatation for the entire follow-up period. The individual dose was adjusted to the largest dose that was clinically tolerated (maximum, $2 \mathrm{mg} / \mathrm{kg}$ body weight). No follow-up investigation was possible in 14 of 46 patients because 2 had an aortic root replacement operation shortly after the initial examination, 9 were examined for the first time less than 1 year before data acquisition for this study was completed (our protocol prescribed yearly intervals), 2 patients had moved and were followed up by other institutions thereafter, and 1 patient refused to participate in the study. In all 14, information about clinical course and therapy was obtained from the attending physician or patient.

According to our protocol, data were obtained before and during $\beta$-blocker treatment at $18 \pm 7$ months (early follow-up) and $39 \pm 16$ months (late follow-up) after the initial investigation. In patients who had an aortic root replacement operation $(n=6 ; 4$ with follow-up), the last preoperative investigation was taken as late follow-up in order not to include investigations of tube grafts and of aortic segments, which are influenced by prosthetic mate- rial. The mean age at late follow-up was $19.9 \pm 11.0$ years. Forty-six age- and sex-matched healthy subjects (some of them were healthy relatives of patients with MFS) constituted the control group and were examined once. Clinical characteristics of patients and controls are shown in Table 1. The protocol was approved by the institutional review board on human research. Written informed consent was obtained from all individuals.

\section{Echocardiographic Evaluation and Calculation of Aortic Parameters}

All subjects underwent a complete transthoracic echocardiographic examination by using commercially available equipment (System Five [General Electric Vingmed Ultrasound, Horten, Norway] or Acuson Sequoia C512 [Siemens Medical Solutions, Mountain View, Calif]). Two-dimensional guided M-mode measurements of the left ventricle were performed according to the recommendations of the American Society of Echocardiography. ${ }^{18}$ Anatomy and function of the mitral and aortic valve were assessed by 2-dimensional echocardiography, color flow mapping, and pulsed-wave and continuous-wave Doppler recordings. We estimated the presence of mitral valve prolapse by using established guidelines. ${ }^{19}$ M-mode tracings of the ascending (AscAo) and descending (DescAo) aortic wall motions were obtained in the left decubitus position according to published criteria ${ }^{20}$ by using $2-$ dimensional guidance at 4 different levels, as described. ${ }^{12,21,22}$ : level 1, annulus (parasternal short-axis view); level 2, sinuses of Valsalva; level 3, proximal AscAo 10 to $20 \mathrm{~mm}$ (in infants, 5 to 10 $\mathrm{mm}$ ) distal to the sinotubular junction (both parasternal long-axis views); and level 4, descending abdominal aorta just proximal to the branching of the celiac trunk (abdominal paramedian long-axis view). Accurate imaging of AscAo and DescAo was achieved in all patients and controls. All images were digitally stored as raw data with EchoPAC System version 6.4.1 (General Electric). Aortic dilatation was determined with standard nomograms. ${ }^{20}$

We calculated aortic diameters continuously at levels 3 and 4 by using software developed by 2 of us (C.B. and D.B.). ${ }^{12,21}$ Averaged time-diameter curves of at least 4 heart cycles and averaged threefold supine systolic and diastolic blood pressure measurements, which were taken according to the recommendations of the American Heart Association ${ }^{23}$ at the right arm oscillometrically (Dinamap; GE Healthcare, Slough, UK) immediately before M-mode registration, served for the automatic calculation of aortic elastic parameters. The method was validated and tested for its reproducibility, as described. ${ }^{12}$ A detailed description of parameters, namely, systolic diameter increase, distensibility, stiffness index, ${ }^{24}$ maximum systolic area increase (MSAI), vector aortographic magnitude, and phase, is given in Appendix 1.

\section{Data Analysis}

Hierarchical cluster analysis. MFS patients were clustered into meaningful subgroups by taking solely aortic parameters into account. Each generated cluster thus represents a characteristic pattern of aortic abnormality based on the similarity of its aggregate expressions (Hierarchical Clustering Explorer 2.0, University of Maryland, College Park, Md).

Logistic regression model. Aortic parameters, selected by a forward search strategy, were used as the input to a logistic regression model. Here, a probability measure in the form of 
TABLE 1. Clinical characteristics and aortic parameters of patients with Marfan syndrome (MFS) and control persons

\begin{tabular}{|c|c|c|c|}
\hline Variable & MFS & Controls & $P$ value \\
\hline No. patients & 46 & 46 & \\
\hline Age (y) & $17.4 \pm 11.1$ & $17.6 \pm 11.1$ & NS \\
\hline Weight (kg) & $51.6 \pm 20.6$ & $50.2 \pm 21.7$ & NS \\
\hline Height $(\mathrm{cm})$ & $166.2 \pm 28.0$ & $155.6 \pm 28.3$ & NS \\
\hline $\operatorname{BSA}\left(\mathrm{m}^{2}\right)$ & $1.54 \pm 0.46$ & $1.47 \pm 0.45$ & NS \\
\hline Heart rate (/min) & $70.2 \pm 18.7$ & $74.0 \pm 21.9$ & NS \\
\hline Systolic blood pressure (mm Hg) & $115.0 \pm 12.2$ & $116.1 \pm 11.9$ & NS \\
\hline Diastolic blood pressure $(\mathrm{mm} \mathrm{Hg})$ & $62.8 \pm 10.9$ & $64.3 \pm 8.7$ & NS \\
\hline Pulse pressure (mm Hg) & $52.2 \pm 10.6$ & $51.8 \pm 10.1$ & NS \\
\hline \multicolumn{4}{|l|}{ Aortic root } \\
\hline Diastolic diameter (mm) & $36.4 \pm 11.7$ & $26.0 \pm 6.7$ & $<.001$ \\
\hline Diastolic diameter/BSA $\left(\mathrm{mm} / \mathrm{m}^{2}\right)$ & $24.8 \pm 6.7$ & $18.7 \pm 4.7$ & $<.001$ \\
\hline \multicolumn{4}{|l|}{ Ascending aorta } \\
\hline Diastolic diameter (mm) & $26.7 \pm 9.7$ & $21.3 \pm 6.1$ & .001 \\
\hline Diameter/BSA $\left(\mathrm{mm} / \mathrm{m}^{2}\right)$ & $18.0 \pm 4.7$ & $15.3 \pm 4.0$ & .001 \\
\hline Systolic diameter increase (\%) & $9.2 \pm 8.6$ & $18.8 \pm 7.3$ & $<.001$ \\
\hline Distensibility $\left(\mathrm{kPa}^{-1} \cdot 10^{-3}\right)$ & $32.8 \pm 23.2$ & $61.1 \pm 25.8$ & $<.001$ \\
\hline Stiffness index & $9.1 \pm 7.3$ & $3.6 \pm 1.3$ & $<.001$ \\
\hline Maximum systolic area increase (\%/100 ms) & $28.1 \pm 16.4$ & $53.7 \pm 25.4$ & $<.001$ \\
\hline \multicolumn{4}{|l|}{ Descending aorta } \\
\hline Diastolic diameter (mm) & $13.7 \pm 5.1$ & $12.6 \pm 3.3$ & NS \\
\hline Diameter/BSA $\left(\mathrm{mm} / \mathrm{m}^{2}\right)$ & $9.2 \pm 2.8$ & $9.1 \pm 2.5$ & NS \\
\hline Systolic diameter increase (\%) & $14.9 \pm 6.8$ & $19.1 \pm 6.4$ & .002 \\
\hline Distensibility $\left(\mathrm{kPa}^{-1} \cdot 10^{-3}\right)$ & $48.6 \pm 28.2$ & $63.3 \pm 27.5$ & .004 \\
\hline Stiffness index & $5.5 \pm 4.9$ & $3.5 \pm 1.7$ & .001 \\
\hline Maximum systolic area increase (\%/100 ms) & $46.5 \pm 23.1$ & $52.0 \pm 19.4$ & NS \\
\hline \multicolumn{4}{|l|}{ Vector aortography } \\
\hline Magnitude (\%) & $19.0 \pm 7.0$ & $26.4 \pm 8.0$ & $<.001$ \\
\hline Phase $\left({ }^{\circ}\right)$ & $61.0 \pm 31.6$ & $46.4 \pm 12.8$ & .009 \\
\hline
\end{tabular}

$B S A$, Body-surface area; NS, not significant. Data are shown as mean \pm SD.

$$
P(z=1)=1 /[1+\exp (-z)],
$$

where $z=a_{O}+a_{1} x_{1}+a_{2} x_{2}+\ldots+a_{n} x_{n}$, is the logit of the model, determines the class membership. $P($ MFS $=1)$ means that MFS is present if $P \geq .5$ and that MFS is absent if $P<.5$. To evaluate follow-up data, the meaning of the dependent variable $P(\mathrm{MFS}=$ $1)$ was changed to $P($ aortic abnormality $=1)$. Fivefold crossvalidation was applied to validate the experimental results (WEKA 3.4.4, University of Waikato, Hamilton, New Zealand).

Statistical analysis. Data are expressed as mean \pm SD. Unpaired continuous variables were compared by means of unpaired Student $t$ tests and Mann-Whitney $U$ tests, respectively. For comparisons of more than 2 variables, the H-test (Kruskal-Wallis) was performed. Paired variables were compared by using the paired $t$ test or Wilcoxon test (SPSS 11.0; SPSS Inc, Chicago, Ill).

\section{Results}

\section{Clinical Characteristics}

Clinical characteristics of the Marfan patients and control persons are summarized in Table 1. Thirty-four patients (74\%) had echocardiographic signs of mitral valve prolapse. Systolic left ventricular function, indicated by fractional shortening, was normal in all patients $(34.0 \% \pm 4.3 \%)$ and controls. In 43 patients $(93 \%)$ dilatation of the aortic root was identified, and in 19 (41\%), dilatation of the AscAo was identified. Thirteen patients $(28 \%)$ presented with aortic regurgitation ( 9 grade $\mathrm{I}+, 2$ grade $\mathrm{II}+$, and 2 grade $\mathrm{III}+$ ), and $29(63 \%)$ presented with mitral regurgitation (14 grade $\mathrm{I}+, 13$ grade $\mathrm{II}+$, and 2 grade III + ), as defined by colorcoded Doppler echocardiography. We did not observe worsening of aortic or mitral regurgitation during follow-up. Thirty patients $(65 \%)$ had a family history of MFS. Mutation analysis of FBN1 in 26 patients revealed a substitution/ missense mutation in 8 patients, a substitution/nonsense mutation in 6 , a deletion/frameshift + premature termination codon mutation in 6 , an indel mutation in 1 , a splice site mutation in 3 , and no mutation in 2 patients. Two siblings showed a mutation of TGFBR2. All mutations but 1 were new, unpublished ones. Details are available upon request.

\section{Aortic Dimensions and Calculation of Elastic Parameters}

Echocardiographic aortic findings of the Marfan group and the control group are shown in Table 1. In patients, AscAo 


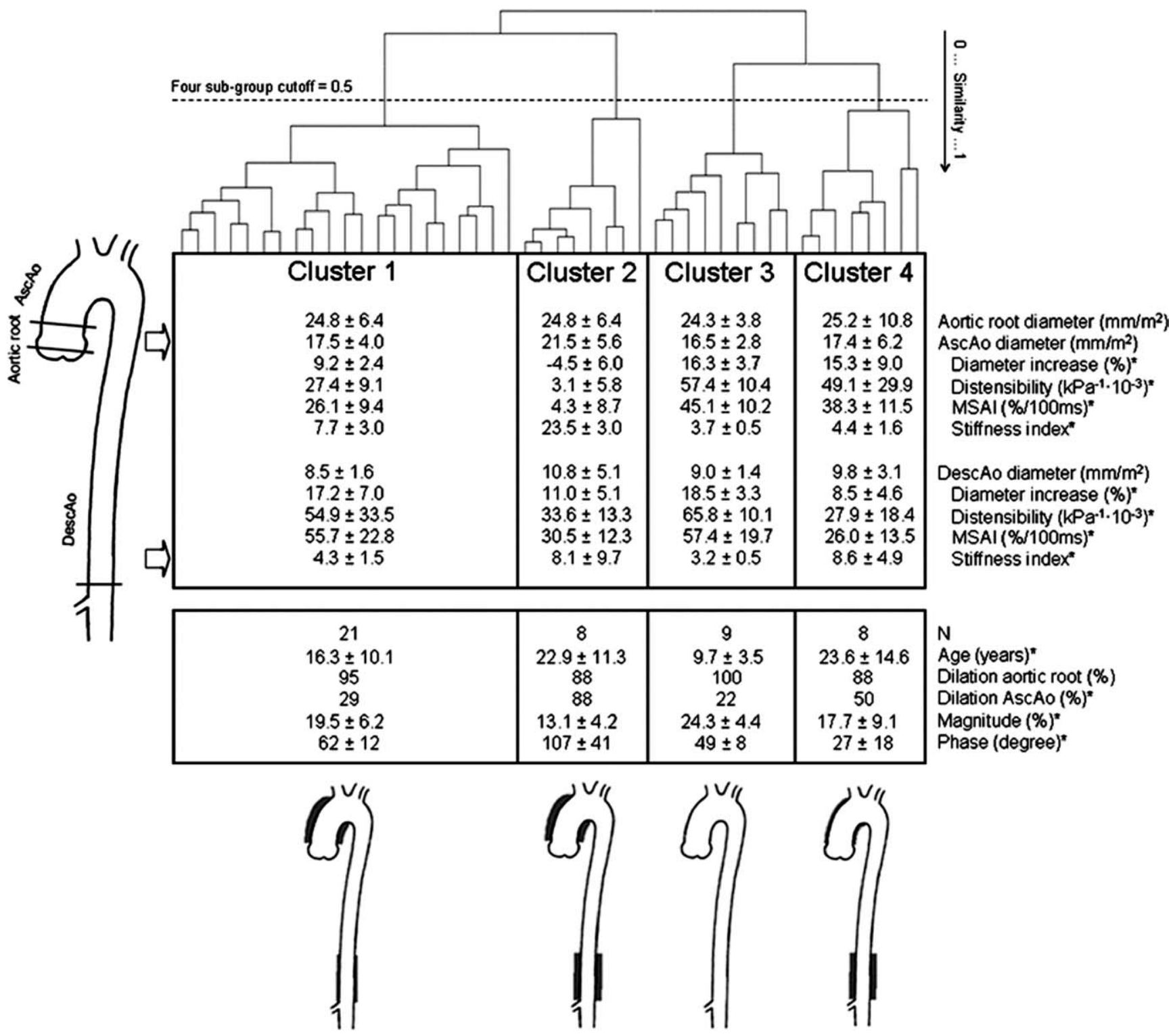

Figure 1. Hierarchical cluster analysis of diameters and elastic parameters of the AscAo, DescAo, and aortic root in 46 MFS patients revealed-at a similarity cutoff of $0.5-4$ clusters representing 4 different aortic patterns of MFS (upper box). Age, percentage of aortic dilatation, vector aortographic magnitude, and phase, which were calculated from clustering results, were different between clusters (lower box). *Aortic parameters significantly different among all 4 clusters $(P<.05)$. Aortic schemes for each cluster show severe (thick lines) or mild (thin lines) AscAo and DescAo wall alterations. Family members sharing the same mutation were spread over different clusters.

and DescAo elastic parameters, such as systolic diameter increase and cross-sectional distensibility, were significantly decreased, whereas the stiffness index was increased compared with healthy controls. MSAI was reduced in the AscAo of patients, but not in the DescAo. Linear regression analysis showed a close correlation between distensibility and MSAI in the AscAo and DescAo of patients $(P=.001$; $r=0.815)$. The vector aortographic magnitude of patients was diminished, and phase was significantly increased, thus indicating a more severe impairment of the AscAo than DescAo systolic diameter increase.

\section{Cluster Analysis}

Because of the phenotypic variability of aortic wall alterations, we performed a hierarchical cluster analysis of AscAo and DescAo parameters in all 46 patients to form 
TABLE 2. Clinical characteristics and aortic diameters of Marfan patients during follow-up

\begin{tabular}{|c|c|c|c|}
\hline Variable & First investigation & Last investigation & $P$ value \\
\hline \multicolumn{4}{|l|}{ Patient characteristics } \\
\hline $\mathrm{n}$ & 30 & 30 & \\
\hline Months of follow-up & 0 & $39 \pm 16$ & \\
\hline Beta-blocker dose $\left(\mathrm{mg} \cdot \mathrm{kg}^{-1} \cdot \mathrm{d}^{-1}\right)$ & 0 & $0.79 \pm 0.46$ & \\
\hline Heart rate (/min) & $70 \pm 22$ & $57 \pm 11$ & $<.001$ \\
\hline Systolic blood pressure (mm Hg) & $114 \pm 12$ & $111 \pm 10$ & NS \\
\hline Diastolic blood pressure (mm Hg) & $64 \pm 10$ & $60 \pm 9$ & .036 \\
\hline \multicolumn{4}{|l|}{ Diameters } \\
\hline Aortic root (mm) & $34.8 \pm 8.9$ & $36.2 \pm 8.4$ & .001 \\
\hline Aortic root/BSA $\left(\mathrm{mm} / \mathrm{m}^{2}\right)$ & $24.5 \pm 6.9$ & $21.9 \pm 4.1$ & .003 \\
\hline AscAo (mm) & $25.9 \pm 8.4$ & $26.8 \pm 7.5$ & .048 \\
\hline AscAo/BSA $\left(\mathrm{mm} / \mathrm{m}^{2}\right)$ & $18.0 \pm 4.6$ & $16.3 \pm 3.9$ & .004 \\
\hline DescAo (mm) & $12.7 \pm 3.6$ & $14.3 \pm 3.5$ & $<.001$ \\
\hline DescAo/BSA $\left(\mathrm{mm} / \mathrm{m}^{2}\right)$ & $8.8 \pm 2.2$ & $8.6 \pm 1.4$ & NS \\
\hline
\end{tabular}

$A s c A o$, Ascending aorta; BSA, body-surface area; DescAo, descending aorta; NS, not significant. Data are mean \pm SD.

meaningful subgroups. The following parameters were included (Figure 1): normalized aortic root, AscAo and DescAo diameters, AscAo and DescAo diameter increase, distensibility, MSAI, and wall stiffness index. Four clusters emerged: Cluster $1(\mathrm{n}=21)$ showed decreased AscAo elasticity. Cluster $2(n=8)$ consisted of patients with severely diminished AscAo and DescAo elastic properties, whereas Cluster $3(\mathrm{n}=9)$ showed very mild AscAo and DescAo alterations in a very young patient group (mean age, 9.7 years vs $17.6 \pm 10.9$ years in controls) not significantly different from those of healthy controls. Nevertheless, dilatation of the aortic root was present in all 9 cases. Cluster $4(\mathrm{n}=8)$ consisted of patients with severely reduced DescAo elasticity accompanied by mild AscAo alterations not significantly different from those of controls. Sixteen of 21 patients in cluster 1,3 of 8 patients in cluster 2, 6 of 9 in cluster 3 , and 7 of 8 in cluster 4 had follow-up investigations of aortic parameters. Notably, $3(37.5 \%)$ of 8 patients of cluster 2 had an aortic root replacement operation after the latest investigation, whereas in clusters 1,3 , and 4 , root replacement was necessary in only $9.5 \%(\mathrm{n}=2)$, $0 \%$, and $12.5 \%(\mathrm{n}=1)$ of patients.

\section{Logistic Regression Models}

Aortic parameters were tested to distinguish between MFS patients and healthy controls. A cross-validated multiple logistic regression model built on 46 patients and 46 controls, including 4 parameters (normalized aortic root and AscAo diameters; AscAo and DescAo distensibility), discriminated with the highest sensitivity $(85.1 \%)$, specificity (87.2\%), and overall accuracy (correctly classified individuals of both groups/all individuals of both groups; $86.2 \%$ ) between the 2 groups. The mean probability $P$ (presence of MFS) was $81.6 \% \pm 24 \%$ for the MFS group and $18.4 \% \pm$ $22.6 \%$ for the control group. Model coefficients, signifi- cance of parameters, and odds ratios are displayed in Appendix 1.

\section{Follow-up Investigation}

Absolute diameters increased in the aortic root $(P=.001)$, the AscAo $(P=.048)$, and the DescAo $(P<.001)$, whereas diameters normalized to body-surface area decreased in the aortic root $(P=.003)$ and the AscAo $(P=.004)$ and remained unchanged in the DescAo (Table 2). AscAo and DescAo elastic parameters (diameter increase, distensibility, stiffness index, magnitude, and phase) tended to improve, and MSAI tended to decrease, but differences were not statistically significant.

To determine the severity of aortic wall disease during follow-up, data were sorted according to aortic root diameter and AscAo distensibility (Figure 2). We therefore interpreted the probability $P$ of our multiple logistic regression model, which included solely aortic parameters, as a measure of aortic abnormality. The overall $P$ (aortic abnormality) of 30 patients decreased significantly from $78 \% \pm$ $27 \%$ to $68 \% \pm 34 \%$ at early follow-up $(P=.01)$ and thereafter remained unchanged $(69 \% \pm 32 \%)$ at late followup. In 21 (70\%) of 30 MFS patients, $P$ (aortic abnormality) decreased from $79 \% \pm 28 \%$ to $63 \% \pm 34 \%$ after $18 \pm 4$ months $(P=.003)$ and remained at $58 \% \pm 33 \%(-21 \%)$ at the last investigation after $41 \pm 16$ months of follow-up and so indicated improvement of elasticity. In the remaining 9 patients $(30 \%), P$ (aortic abnormality) changed insignificantly from $75 \% \pm 24 \%$ to $77 \% \pm 35 \%$ after $18 \pm 11$ months but increased further to $93 \% \pm 6 \%$ after $31 \pm 16$ months of follow-up $(+18 \% ; P=.05)$, as a sign of deterioration of aortic wall mechanics. Neither the mean follow-up time $(P=.164)$ nor the mean $\beta$-blocker dosage $(0.78 \pm$ $0.50 \mathrm{mg} \cdot \mathrm{kg}^{-1} \cdot \mathrm{d}^{-1}$ vs $0.82 \pm 0.36 \mathrm{mg} \cdot \mathrm{kg}^{-1} \cdot \mathrm{d}^{-1} ; P=$ .756 ) of the 2 groups was statistically different. $P$ (aortic 


Aortic root diameter
Age examination 1
B-blocker dose

20 - $30 \mathrm{~mm}$

$5.3 \pm 4.2$ years

$0.68 \pm 0.59 \mathrm{mg} / \mathrm{kg}$

$\mathrm{N}=7$
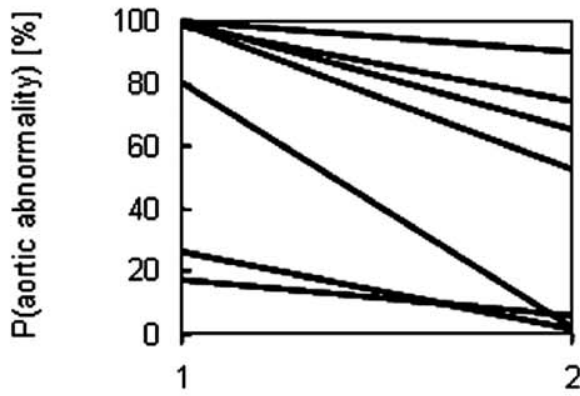

A

$$
\begin{gathered}
P=75 \pm 37 \% \rightarrow P=42 \pm 38 \% \\
\downarrow 100 \% \text { of cases }
\end{gathered}
$$

\section{AscAo distensibility \\ Age examination 1 \\ B-blocker dose \\ $83-\mathbf{4 5} \mathbf{~ P P a}^{-1 \cdot 10^{-3}}$
$10.4 \pm 5.4$ years
$0.95 \pm 0.63 \mathrm{mg} / \mathrm{kg}$ \\ $\mathrm{N}=8$}

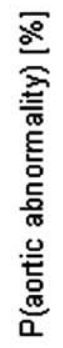

B

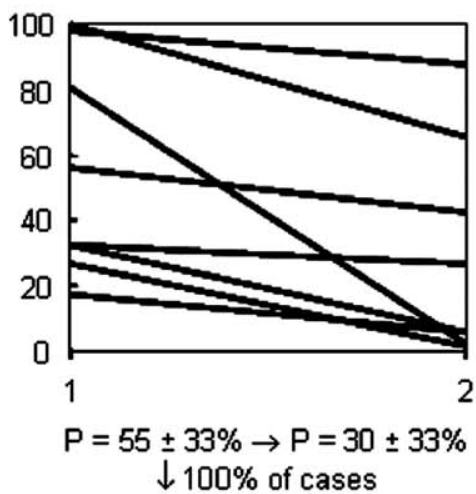

$30-\mathbf{4 0} \mathrm{mm}$

$14.5 \pm 4.4$ years

$0.80 \pm 0.4 \mathrm{mg} / \mathrm{kg}$

$\mathrm{N}=13$

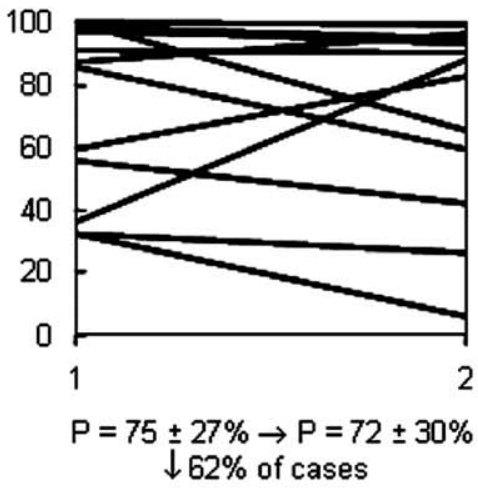

$45-30 \mathrm{kPa}^{-1} \cdot 10^{-3}$

$17.6 \pm 11.1$ years

$0.68 \pm 0.37 \mathrm{mg} / \mathrm{kg}$

$\mathrm{N}=12$

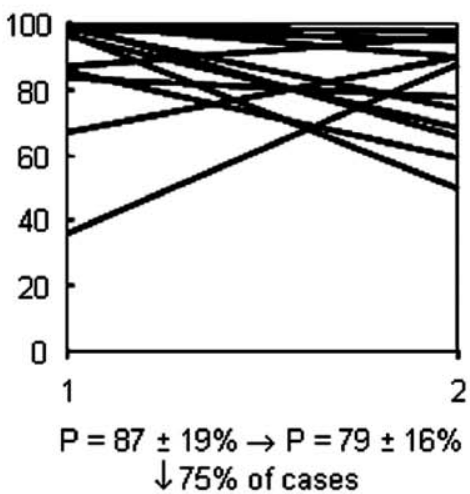

$40-52 \mathrm{~mm}$

$28.3 \pm 10.0$ years

$0.87 \pm 0.44 \mathrm{mg} / \mathrm{kg}$

$\mathrm{N}=10$

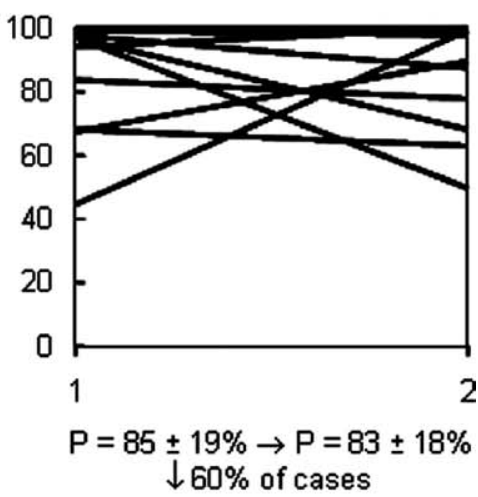

30 - $0 \mathrm{kPa}^{-1} \cdot 10^{-3}$

$21.4 \pm 12.6$ years

$0.80 \pm 0.37 \mathrm{mg} / \mathrm{kg}$

$\mathrm{N}=10$

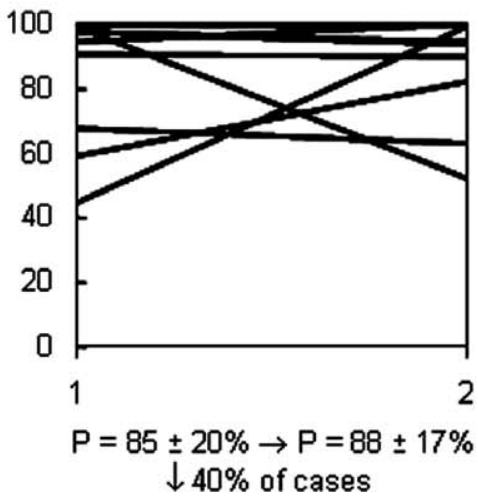

Figure 2. Probability P(aortic abnormality) at the initial (1) and final (2) investigation in 30 MFS patients sorted for the diameter of the aortic root $(A)$ and AscAo distensibility (B). Note the improvement of $P$ (aortic abnormality) of all patients with root diameters less than $30 \mathrm{~mm}$ and/or AscAo distensibility greater than $45 \mathrm{kPa}^{-1} \cdot 10^{-3}$.

abnormality) decreased in all patients with aortic root diameters between 20 and $30 \mathrm{~mm}$ (Figure 2, $A$; left subgroup), in $62 \%$ with diameters between 30 and $40 \mathrm{~mm}$ (middle), and in $60 \%$ with diameters between 40 and $52 \mathrm{~mm}$ (right).

If data were sorted according to AscAo distensibility (Figure $2, B$ ), the probability $P$ (aortic abnormality) decreased in $100 \%$ of patients with an AscAo distensibility between 45 and $83 \mathrm{kPa}^{-1} \cdot 10^{-3}$, in $75 \%$ of patients with a distensibility between 30 and $45 \mathrm{kPa}^{-1} \cdot 10^{-3}$, and in $40 \%$ with a distensibility between 0 and $30 \mathrm{kPa}^{-1} \cdot 10^{-3}$. The $\beta$-blocker dose did not differ significantly among these 3 subgroups (H-test).

The pattern of aortic abnormality did not change during follow-up, because the vector aortographic maximum magnitude and phase of clusters remained unchanged. Solely in cluster 1 with predominant AscAo elasticity impairment (n $=16), P($ aortic abnormality) decreased and, therefore, elasticity improved at the late follow-up investigation, explicitly from $85.2 \% \pm 22.0 \%$ to $69.5 \% \pm 27.8 \%(-15.6 \% ; P=$ .027). The mean $P$ (aortic abnormality) values of clusters 2 (initially $97.2 \%)$, cluster $3(53.4 \%)$, and cluster 4 (78.6\%) remained unchanged.

Not surprisingly, the youngest patients were found in the groups with the smallest diameters and the greatest distensibility, whereas in both patients who had not received $\beta$-blockers (11 and 31 years old), $P$ (aortic abnormality) increased (from $55.6 \%$ to $79.8 \%$ and from $54.2 \%$ to $89.3 \%$, respectively). All patients who had undergone aortic root replacement operation after our examinations belonged to a subgroup with aortic root diameters greater than $44 \mathrm{~mm}$ and 
AscAo distensibility between 0 and $27 \mathrm{kPa}^{-1} \cdot 10^{-3}$ at the initial investigation (Figure 2, right subgroup). Their initial $P$ (aortic abnormality) ranged between $68 \%$ and $100 \%$ (median, 99\%) and improved in 2 of 4 cases at late follow-up during $\beta$-blocker treatment.

\section{Discussion}

Our study demonstrated that aortic wall changes of patients with MFS followed 4 different patterns: involvement of AscAo (46\% of our patients), severe AscAo and DescAo changes (17\%), mild AscAo and DescAo changes not significantly different from normal (20\%), and dominant DescAo involvement (17\%). One-half of the patients (3 of 6) who had an elective aortic root replacement operation after our examinations were in cluster 2, with severe AscAo and DescAo wall alterations; none were in cluster 3, with mild AscAo and DescAo changes. This means that 3 of 8 patients in cluster 2 had to be operated on on the basis of conventional criteria for surgical intervention. Therefore, cluster 2 might be regarded as a risk group for aortic complications; in cluster 3, the risk should be lowest. Histologic examinations in patients with MFS revealed that elastin fragmentation in the aortic media is scattered in an irregular pattern along the aorta. ${ }^{25}$ Investigations of patients with fusiform AscAo aneurysms disclosed media degeneration of the AscAo wall. ${ }^{26}$ Here, the predominance of AscAo changes probably reflects the ratio of elastic fiber to collagen, which varies from 3.1:1 in the proximal AscAo to 2.8:1 in the midthoracic region and 0.8:1 in the abdominal region. ${ }^{11,27}$ In a study of 192 patients with MFS who underwent aortic aneurysm repair, $16 \%$ had their initial operation for aortic dissection in the DescAo. ${ }^{28}$ We speculate that our subgroup with DescAo changes perhaps is at risk for DescAo complications.

It is well known that degenerative aortic changes in Marfan patients parallel alterations due to normal aging, which can be measured in terms of arterial stiffness. ${ }^{29}$ Mild AscAo and DescAo changes not significantly different from normal controls were seen in our youngest patient subgroup (cluster 3). Older patients were subdivided into 3 subgroups with either (1) AscAo, (2) severe AscAo and DescAo, or (3) merely DescAo changes. In family members sharing the same mutation, different aortic patterns were observed. During a follow-up time of 39 months, our patients did not change their pattern of aortic involvement, which underlines the results of a study which showed that local initial diameters were the major predictors of progressive aortic root and abdominal aortic dilatation. ${ }^{11}$ Longer follow-up studies may show the change from insignificant to severe aortic alterations.

The 4 key features determining aortic abnormality in our multiple logistic regression model were the following: aortic root and AscAo diameters normalized to body-surface area and AscAo and DescAo distensibility. The model distinguished with high sensitivity (85\%) and specificity (87\%) between MFS patients and healthy control subjects. Because of its suitability, this model was successfully introduced into our daily clinical practice.

Probability measures resulting from logistic regression analysis clearly quantify clinical situations determined by multiple factors and, thereby, can indicate improvement or deterioration during follow-up. M-mode registration at exactly the same aortic sites, which is essential for comparison of results, can be performed accurately by our method. Our model revealed that $P$ (aortic abnormality) decreased and therefore showed improvement in 100\% of patients with aortic root diameters less than $30 \mathrm{~mm}$ during follow-up and treatment with $\beta$-blockers, whereas improvement occurred only in approximately $60 \%$ of patients with root diameters greater than $30 \mathrm{~mm}$. In a study of 13 adult patients with MFS, ${ }^{15} 8$ responded to a single dose of metoprolol with a decrease of stiffness index (responders). The group of the 5 nonresponders was characterized by an increasing stiffness index and more dilated AscAo. In analogy, 100\% of our patients with an AscAo distensibility more than $45 \mathrm{kPa}^{-1}$. $10^{-3}$ improved, $75 \%$ with a distensibility between 30 and $45 \mathrm{kPa}^{-1} \cdot 10^{-3}$ improved, and only $40 \%$ with a distensibility less than $30 \mathrm{kPa}^{-1} \cdot 10^{-3}$ improved. Improvement could already be observed after 18 months of $\beta$-blocker treatment, whereas deterioration occurred gradually and reached significance at late follow-up. It is interesting to note that only the cluster 1 patient group with AscAo wall changes showed a positive response to $\beta$-blocker treatment, indicated by an improvement of $P$ (aortic abnormality). The smallness of clusters 2 to 4 has to be taken into account, and results must be viewed cautiously.

Our study does have a limitation. The descending thoracic aorta cannot be examined by our investigation method. This should regularly be done by additional cine-magnetic resonance imaging.

A recent prospective study in a mouse model of aortic aneurysm provides a newer understanding of many aspects of MFS that were shown to be caused by dysregulation and enhanced signaling of the transforming growth factor $\beta$ family of cytokines. ${ }^{30}$ Fibrillin-1 shares a high degree of homology with the latent transforming growth factor $\beta$-binding proteins. Aortic aneurysm in this study could be prevented by transforming growth factor $\beta$ antagonists such as transforming growth factor $\beta$-neutralizing antibody or the angiotensin II type 1 receptor blocker losartan, a drug already in clinical use for hypertension. Our logistic regression model could perhaps be a useful tool to prove these promising results in a randomized controlled trial of losartan and $\beta$-blockers in humans.

In summary, our multiple logistic regression model including solely AscAo and DescAo parameters is a sensitive 
tool to determine the probability of MFS. If $P$ (aortic abnormality) is high, the similarity to 1 of the 4 clusters probably shows the aortic region to be at risk for complications. Follow-up investigations during pharmacologic therapy can monitor its success. We observed improvement of aortic wall changes in patients with predominant AscAo wall abnormality, in all (young) patients with aortic root diameters less than $30 \mathrm{~mm}$, and in those with AscAo distensibility more than $45 \mathrm{kPa}^{-1} \cdot 10^{-3}$. These findings underscore the usefulness of early pharmacologic therapy. Finally, an increase of $P$ (aortic abnormality) can be used as additional argument to indicate prophylactic surgical aortic intervention.

\section{References}

1. Pyeritz RE, McKusick VA. The Marfan syndrome: diagnosis and management. N Engl J Med. 1979;300:772-7.

2. Judge DP, Dietz HC. Marfan's syndrome. Lancet. 2005;366:1965-76.

3. Murdoch JL, Walker BA, Halpern BL, Kuzma JW, McKusick VA. Life expectancy and causes of death in the Marfan syndrome. $N$ Engl J Med. 1972;286:804-8.

4. Engelfriet PM, Boersma E, Tijssen JG, Bouma BJ, Mulder BJM. Beyond the root: dilatation of the distal aorta in the Marfan syndrome. Heart. Feb 17, 2006 [Epub ahead of print].

5. Yin FCP, Brin KP, Ting CT, Pyeritz RE. Arterial hemodynamic indexes in Marfan's syndrome. Circulation. 1989;79:854-62.

6. Hirata K, Triposkiadis F, Sparks E, Bowen J, Wooley CF, Boudoulas $\mathrm{H}$. The Marfan syndrome: abnormal aortic elastic properties. J Am Coll Cardiol. 1991;18:57-63.

7. Savolainen A, Keto P, Hekali P, Nisula L, Kaitila I, Vitasalo M, et al. Aortic distensibility in children with the Marfan syndrome. Am J Cardiol. 1992;70:691-3.

8. Adams JN, Brooks M, Redpath TW, Smith FW, Dean J, Gray J, et al. Aortic distensibility and stiffness index measured by magnetic resonance imaging in patients with Marfan's syndrome. Br Heart J. 1995; 73:265-9.

9. Groenink M, de Roos A, Mulder BJM, Spaan JAE, van der Wall EE Changes in aortic distensibility and pulse wave velocity assessed with magnetic resonance imaging following beta-blocker therapy in the Marfan syndrome. Am J Cardiol. 1998;82:203-8.

10. Jondeau G, Boutouyrie P, Lacolley P, Laloux B, Dubourg O, Bourdarias JP, et al. Central pulse pressure is a major determinant of ascending aorta dilation in Marfan syndrome. Circulation. 1999;99: 2677-81.

11. Nollen GJ, Groenink M, Tijssen JGP, van der Wall EE, Mulder BJM. Aortic stiffness and diameter predict progressive aortic dilatation in patients with Marfan syndrome. Eur Heart J. 2004;25:1146-52.

12. Baumgartner D, Baumgartner C, Matyas G, Steinmann B, Löffler J, Schermer E, et al. Diagnostic power of aortic elastic properties in young patients with Marfan-syndrome. J Thorac Cardiovasc Surg. 2005;129:730-9.

13. Shores J, Berger KR, Murphy EA, Pyeritz RE. Progression of aortic dilatation and the benefit of long-term $\beta$-adrenergic blockade in Marfan's syndrome. N Engl J Med. 1994;330:1335-41.

14. Salim MA, Alpert BS, Ward JC, Pyeritz RE. Effect of beta-adrenergic blockade on aortic root rate of dilation in the Marfan syndrome. Am J Cardiol. 1994;74:629-33.

15. Haouzi A, Berglund H, Pelikan PCD, Maurer G, Siegel RJ. Heterogeneous aortic response to acute $\beta$-adrenergic blockade in Marfan syndrome. Am Heart J. 1997;133:60-3.

16. De Paepe A, Devereux RB, Dietz HC, Hennekam RCM, Pyeritz RE. Revised diagnostic criteria for the Marfan syndrome. Am J Med Genet. 1996;62:417-26.

17. Mátyás G, De Paepe A, Halliday D, Boileau C, Pals G, Steinmann B. Evaluation and application of denaturing HPLC for mutation detection in Marfan syndrome: identification of 20 novel mutations and two novel polymorphisms in the FBN1 gene. Hum Mutat. 2002;19:443-56.

18. Sahn DJ, DeMaria A, Kissolo J, Weyman A. Recommendations regarding quantitation in M-mode echocardiography: results of a survey of echocardiographic measurements. Circulation. 1978;58:1072-83.

19. Devereux RB, Kramer-Fox R, Shear MK, Kligfield P, Pini R, Savage DD. Diagnosis and classification of severity of mitral valve prolapse: methodologic, biologic, and prognostic considerations. Am Heart $J$. 1987;113:1265-80.

20. Roman MJ, Devereux RB, Kramer-Fox R, O'Loughlin J. Two-dimensional echocardiographic aortic root dimensions in normal children and adults. Am J Cardiol. 1989;64:507-12.

21. Baumgartner C, Mátyás G, Steinmann B, Baumgartner D. Marfan syndrome: a diagnostic challenge caused by phenotypic and genetic heterogeneity. Methods Inf Med. 2005;44:487-97.

22. Vogt M, Kühn A, Baumgartner D, Baumgartner C, Busch R, Kostolny $\mathrm{M}$, et al. Impaired elastic properties of the ascending aorta in newborns before and early after successful coarctation repair: proof of a systemic vascular disease of the prestenotic arteries? Circulation. 2005;111: 3269-73.

23. National High Blood Pressure Education Program Working Group on High Blood Pressure in Children and Adolescents. The fourth report on the diagnosis, evaluation, and treatment of high blood pressure in children and adolescents. Pediatrics. 2004;114:555-76.

24. Kawasaki T, Sasayama S, Yagi SI, Asakawa T, Hirai T. Non-invasive assessment of the age related changes in stiffness of major branches of the human arteries. Cardiovasc Res. 1987;21:678-87.

25. Schlatmann TJ, Becker AE. Histologic changes in the normal aging aorta: implications for dissecting aortic aneurysm. Am J Cardiol. 1977;39:13-20.

26. Roberts WC, Honig HS. The spectrum of cardiovascular disease in the Marfan syndrome: a clinico-morphologic study of 18 necropsy patients and comparison to 151 previously reported necropsy patients. Am Heart J. 1982;104:115-35.

27. Virmani R, Avolio AP, Mergner WJ, Robinowitz M, Herderick EE, Cornhill JF, et al. Effect of aging on aortic morphology in populations with high and low prevalence of hypertension and atherosclerosis: comparison between occidental and Chinese communities. Am J Pathol. 1991;139:1119-29.

28. Finkbohner R, Johnston D, Crawford S, Coselli J, Milewicz DM. Marfan syndrome: long-term survival and complications after aortic aneurysm repair. Circulation. 1995;91:728-33.

29. Vlachopoulos C, Aznaouridis K, Stefanadis C. Clinical appraisal of arterial stiffness: the Argonauts in front of the Golden Fleece. Heart. Dec 9, 2005 [Epub ahead of print].

30. Habashi JP, Judge DP, Holm TM, Cohn RD, Loeys BL, Cooper TK, et al. Losartan, an AT1 antagonist, prevents aortic aneurysm in a mouse model of Marfan syndrome. Science. 2006;312:117-21.

\section{Appendix 1: Aortic parameters}

1. Systolic diameter increase:

$$
\left(D_{\mathrm{s}}-D_{\mathrm{d}}\right) / D_{\mathrm{d}}(\%),
$$

where $D_{\mathrm{s}}$ is systolic (maximum) and $D_{\mathrm{d}}$ is end-diastolic (minimum) aortic diameter. The cross-sectional aortic distensibility and stiffness index were estimated as previously described. ${ }^{7,12,21,24}$

2. Distensibility:

$$
\frac{A_{\mathrm{s}}-A_{\mathrm{d}}}{A_{\mathrm{d}} \cdot\left(P_{\mathrm{s}}-P_{\mathrm{d}}\right) \cdot 1333} \cdot 10^{7}\left(\mathrm{kPa}^{-1} \cdot 10^{-3}\right),
$$

3. Stiffness index:

$$
\frac{\ln \left(P_{\mathrm{s}} / P_{\mathrm{d}}\right)}{\left(D_{\mathrm{s}}-D_{\mathrm{d}}\right) / D_{\mathrm{d}}} \text { (dimensionless), }
$$


where $A_{\mathrm{s}}$ is systolic and $A_{\mathrm{d}}$ is end-diastolic area and $P_{\mathrm{s}}$ is systolic and $P_{\mathrm{d}}$ is diastolic blood pressure $(\mathrm{mm} \mathrm{Hg})$. Area $A$ was determined as $(\mathrm{D} / 2)^{2} \cdot \pi$.

4. Maximum systolic area increase (MSAI) was defined as the maximum systolic slope of the area-time curve $A(\mathrm{t})$ normalized to $A_{\mathrm{d}}$ :

$$
\text { MSAI }=\frac{d}{d t}\left(\left(A(\mathrm{t}) / A_{\mathrm{d}}-1\right) \cdot 100\right) \mathrm{|}_{\max }(\% / 100 \mathrm{~ms})
$$

The vector aortography visualizes the vector loop of the relative AscAo (x-axis) and DescAo (y-axis) diameter changes during the heart cycle. The rotating vector can be characterized by its magnitude (vector length) and phase (vector angle).

5. Magnitude (t):

$$
\sqrt{\left[\left(D(\mathrm{t}) / D_{\mathrm{d}}-1\right) \cdot 100\right]_{\mathrm{AscAo}}^{2}+\left[\left(D(\mathrm{t}) / D_{\mathrm{d}}-1\right) \cdot 100\right]_{\text {DescAo }}^{2}}(\%),
$$

6. Phase $(\mathrm{t})$ :

$$
\arctan \left(\frac{\left(D(\mathrm{t}) / D_{\mathrm{d}}-1\right)_{\text {DescAo }}}{\left(D(\mathrm{t}) / D_{\mathrm{d}}-1\right)_{\text {AscAo }}}\right)\left(^{\circ}\right)
$$

\begin{tabular}{|c|c|c|c|c|}
\hline Model parameter & Regression coefficient & SE & $P$ value & Odds ratio \\
\hline Normalized root diameter $\left(\mathrm{mm} / \mathrm{m}^{2}\right)$ & 0.842 & 0.201 & $<.001$ & 2.321 \\
\hline Normalized AscAo diameter $\left(\mathrm{mm} / \mathrm{m}^{2}\right)$ & -0.727 & 0.215 & .001 & 0.483 \\
\hline AscAo distensibility $\left(\mathrm{kPa}^{-1} \cdot 10^{-3}\right)$ & -0.098 & 0.027 & $<.001$ & 0.907 \\
\hline DescAo distensibility $\left(\mathrm{kPa}^{-1} \cdot 10^{-3}\right)$ & -0.033 & 0.015 & .028 & 0.968 \\
\hline Intercept & 0.352 & 1.795 & .844 & 1.422 \\
\hline
\end{tabular}

where $D(\mathrm{t})$ is the aortic diameter/time curve.

\section{Logistic regression model}

AscAo, Ascending aorta; DescAo, descending aorta. 